\title{
Cycloops: Dark Matter or a Monopole Problem for Brane Inflation?
}

\author{
A. Avgoustidi.* and E.P.S. Shellard \\ Department of Applied Mathematics and Theoretical \\ Physics, Centre for Mathematical Sciences, \\ University of Cambridge, Wilberforce Road, \\ Cambridge CB3 0WA, United Kingdom
}

\begin{abstract}
We consider cosmic loop production by long string interactions in cosmological models with compact extra dimensions. In the case that the compact manifold is not simply connected, we focus on the possibility of loops wrapping around non-trivial cycles and becoming topologically trapped. Such loops, denoted cycloops, behave like matter in the radiation era, posing a potential monopole problem. We calculate the number distribution and the energy density of these objects as functions of cosmic time and use them to study cosmological constraints imposed on simple brane inflation models. For typical choices of parameters we find that to avoid cycloop domination before the matter-radiation transition, the strings must be unacceptably light, namely $G \mu<10^{-18}$, unless some mechanism to dilute the cycloops is provided. By exploring the full parameter space however, we are able to find models with $G \mu \sim 10^{-14}$, which is marginally consistent with brane inflation. In such models the cycloop could provide an interesting dark matter candidate.
\end{abstract}

Keywords: cosmic loops, extra dimensions, brane inflation, monopoles

*Electronic address: A.Avgoustidis@damtp.cam.ac.uk

$\dagger$ Electronic address: E.P.S.Shellard@damtp.cam.ac.uk 


\section{INTRODUCTION}

There has been a notable revival of interest in cosmic strings recently both for theoretical and observational reasons (for a review see Ref. [1]). The main motivation comes from brane inflation 2, 3], where cosmic strings are produced at the end of an inflationary phase [4, 5], and in particular the proposal that these strings can be fundamental (super)strings (Fstrings), D-strings, or bound states between them [5, 6]. The fact that cosmic strings can be produced as topological defects in brane inflation is particularly exciting because such strings could be observable [7, 8, 9], providing a potentially observational handle on superstring physics [10, 11]. Indeed, cosmic strings appearing in these models can be substantially different than usual strings. They can be very light $\left(10^{-12}<G \mu<10^{-7}\right)$ and can have an intercommuting probability much less than unity [7, 12]. For such strings, interactions are suppressed leading to a much greater string number density today. More complicated 'entangled' networks, with strings joining at trilinear vertices, can also be formed [5, 6].

The evolution of these strings was first discussed in terms of a one-scale model in Ref. 7] (also see Ref. 51). More recently, an extension of the Velocity-dependent-One-Scale (VOS) model [13, 14, 15] for theories with extra dimensions has been presented (the EDVOS model) 16], which can be used for more quantitative estimates of scaling string densities. The evolution of non-intercommuting and entangled networks within a VOS model approximation was studied in [17] (see also [18]). Evolving strings with small intercommuting probability has also been discussed in Refs. [9, 19, 20].

The purpose of this paper is to study the evolution of closed cosmic strings (loops) in the presence of extra compact dimensions. As noted in Ref. [5], if the compact manifold is not simply connected, such loops can wrap around non-trivial cycles and become topologically trapped. These objects will behave like monopoles and could pose constraints on the models which have them. Similar monopole-like objects have also been discussed recently in Refs. 21, 22], but their cosmological evolution or the constraints they impose have not been fully investigated. Further constraints exist on a class of cosmic superstring models, for example from dilaton emission [23].

The paper is organised as follows. In section [I] we briefly review the evolution of cosmic loops in three spatial dimensions and then focus on the case where extra compact dimensions are present. We consider the possibility of loops winding around the extra dimensions and 
show that their evolution can be very different from usual loops, if the compact manifold admits non-trivial 1-cycles. In particular, their energy density scales like matter in the radiation era, posing a potential monopole problem. In section III we consider the cosmological constraints imposed on simple brane inflation models by requiring the absence of such a monopole problem. Our conclusions are summarised in section IV

\section{COSMIC LOOPS}

Numerical simulations of cosmic string formation after symmetry breaking phase transitions suggest that roughly $80 \%$ of the total string length is in the form of infinite Brownian strings, while the remaining $20 \%$ is in closed loops 24], though these ratios depend somewhat on the topology of symmetry breaking (see 25] and references therein). These loops normally oscillate and radiatively decay, but more loops are dynamically produced by long string interactions. Indeed, a string curling back on itself or two colliding strings can chop off one or more small loops. Simulations of string evolution verify this picture, though the evidence for linear scaling of loop sizes is only now emerging relative to the smallest possible size allowed by the numerical resolution [26, 27, 28]. Such loops typically have a very small cross-section and decouple from the long string network, thus taking energy away from it. This mechanism is crucial in ensuring that the network achieves a scaling solution, that is, one in which the correlation length remains constant with respect to the horizon size [25, 29]. In the following we will briefly review how the loop energy density evolves in time in the standard picture and then identify the possibility of distinctively different evolution in the presence of compact extra dimensions.

\section{A. Standard Loops}

The scaling property of the long string network allows one to describe the production of loops in terms of a scale-invariant function $f(\ell / L)[29]$. This is defined so that the energy loss into loops of size between $\ell$ and $\ell+\delta \ell$ per correlation volume per unit time is $\mu f(\ell / L) \delta \ell / L$, where $\mu$ is the cosmic string tension and $L$ the correlation length, defined so that the energy density of the long string network is given by $\rho_{\infty}=\mu / L^{2}$. One then defines a loop number density distribution $n(\ell, t)$ so that $n(\ell, t) \delta \ell$ gives the number density of loops in the length 
range $\ell$ to $\ell+\delta \ell$. A loop energy density distribution can also be defined by

$$
\rho(\ell, t)=\mu \ell n(\ell, t)
$$

Taking into account the dilution due to Hubble expansion, an equation for the rate of change of the loop energy density distribution can be written

$$
\dot{\rho}(\ell, t)=-3\left(\frac{\dot{a}}{a}\right) \rho(\ell, t)+g \frac{\mu}{L^{4}} f(\ell / L)
$$

where $a(t)$ is the scalefactor and $g$ a Lorentz factor accounting for non-zero center-of-mass kinetic energies of the produced loops. Equation (2) can be integrated to give at late times in the radiation era $[25]$

$$
\rho(\ell, t)=\frac{\mu g \gamma^{-5 / 2}}{(t \ell)^{3 / 2}} \int_{0}^{\infty} \sqrt{x} f(x) d x .
$$

where $\gamma$ is defined from $L=\gamma t$ and is constant for a scaling network.

We now use an approximation in which all loops are produced at a constant size relative to the horizon i.e. $\ell=\alpha t$. Then the loop production function becomes a $\delta$-function

$$
f(x)=\tilde{c} \delta(x-\alpha / \gamma)
$$

so that

$$
\nu_{r}=g \gamma^{-3} \alpha^{1 / 2} \tilde{c}
$$

For standard strings the constant $\tilde{c}$ (the loop production parameter) can be extracted from simulations and is of order unity [14]. From equations (11), (3) and (15) we can read the loop number density distribution in this approximation

$$
n(\ell, t)=\frac{g \tilde{c} \gamma^{-3} \alpha^{1 / 2}}{t^{3 / 2} \ell^{5 / 2}}
$$

So far we have assumed that loops have constant length $\ell$. We know however that they lose energy (and hence length) due to radiative processes. Assuming that the dominant decay mechanism is emission of gravitational radiation, a loop of initial size $\ell_{i}$ (formed at time $\left.t_{i}\right)$ will have a length $\ell=\ell_{i}-\Gamma G \mu\left(t-t_{i}\right)$ at a later time $t$, where $\Gamma$ is a constant of order 65 [25]. Since equation (66) is expressed in terms of the length at which the loops are produced, we can write for the number density distribution at time $t$

$$
n(\ell, t)=\frac{g \tilde{c} \gamma^{-3} \alpha^{1 / 2}}{t^{3 / 2}\left[\ell+\Gamma G \mu\left(t-t_{i}\right)\right]^{5 / 2}} .
$$


Because of the $\delta$-function approximation (imposing $\ell_{i}=\alpha t_{i}$ ) all loops of a given length $\ell$ at time $t$ were produced at the same time $t_{i}$ (when they had the same initial length $\alpha t_{i}$ ). For small loops at late times $t>t_{i}$ in (17) but for large loops (of length comparable to $\alpha t$ ) $\ell$ is similar to its initial size $\ell_{i}$ and $t_{i}$ cannot be neglected. The maximum size of loops at time $t$ is $\alpha t$ and corresponds to the loops that have just been created $\left(t_{i}=t\right)$.

The dominant contribution to $n(\ell, t)$ is from the smallest loops so that we can ignore $t_{i}$ in equation (17). For $\ell>\Gamma G \mu t$ the distribution is dominated by small $\ell$, but for $\ell<\Gamma G \mu t$ it is linear in $\ell$, so that the dominant contribution comes from loops of size $\ell \sim \Gamma G \mu t$. We therefore have, by integrating $n(\ell, t)$ over all loop sizes,

$$
\left.n(t) \approx \frac{2}{3} \frac{g \tilde{c} \gamma^{-3} \alpha^{1 / 2}}{(t \ell)^{3 / 2}}\right|_{\ell=\Gamma G \mu t}=\frac{2}{3} \frac{g \tilde{c} \gamma^{-3} \alpha^{1 / 2}}{(\Gamma G \mu)^{3 / 2} t^{3}} .
$$

The loop energy density at time $t$ in the radiation era is similarly

$$
\rho(t) \approx 2\left(\frac{\alpha}{\Gamma G \mu}\right)^{1 / 2} \frac{g \tilde{c} \mu}{\gamma^{3} t^{2}},
$$

which scales like the (radiation) background density. Eventually, matter will dominate and the loops will only contribute a negligible amount to the energy density of the universe.

However, in theories with extra compact dimensions it is possible that loops can wrap around 1-cycles in the compact manifold and become topologically trapped, not being able to shrink to zero size [5]. We call such objects 'cycloops' that is, loops winding around non-trivial cycles. One expects the energy density of these objects to scale like matter so the possibility that they can dominate the universe arises. We consider cycloops in more detail below.

\section{B. Cycloops: a monopole problem for brane inflation}

We now consider the case where the string network evolves in a $(D+1)$-dimensional spacetime with three large FRW spatial dimensions and the rest compactified at a scale $R$, which is less than the correlation length $L$. We can still describe this situation by an effective three dimensional model in which we introduce an intercommuting probability to account for the fact that strings can miss each other, because of the presence of extra dimensions [5, 7, 16]. The discussion in the previous section follows up to equation (6) but now the loop 
production parameter $\tilde{c}$ is suppressed by a factor

$$
P \sim\left(\frac{\delta}{R}\right)^{D-3}
$$

where $\delta$ is the effective string thickness, a capture radius for string interactions (for a calculation of $\mathrm{P}$ in string theory see [12]).

The scaling parameter $\gamma$ is also suppressed by a factor of $P$ within the one-scale approximation [7] but small-scale-structure on strings is expected to lead to a weaker $P$ dependence. This can be incorporated in the one-scale or VOS model by introducing an effective intercommuting probability $P_{\text {eff }}=f(P)[16]$. Flat space simulations suggest that $\gamma$ is suppressed by a factor of $\sqrt{P}$ rather than $P[5,19,20]$ and simulations in expanding space are in progress [30].

Thus, with the understanding that $\tilde{c}$ and $\gamma$ are suppressed compared to their standard three-dimensional values, the number density distribution of loops with respect to their initial length is given by (6). These loops can radiate energy reducing their length, but, unlike the three-dimensional case, not all loops can shrink to zero. If the compact manifold is not simply connected then some loops can wrap around non-trivial 1-cycles and become trapped. These loops, the cycloops, can only shrink down to a minimum size equal to their winding number times the size of the non-trivial cycle. They will therefore look like stable monopoles to a three-dimensional observer. We note that similar objects were recently found in Ref. [21] as a result of D-brane annihilation in the case that the correlation length of the produced brane-defects is smaller than the size of the extra dimensions. Ref. [22] also discusses monopole-like objects arising as kink configurations on the worldvolume of long strings, associated to the scalar fields that describe the string position in the extra dimensions.

Since loops are formed at a length $\alpha$, larger and larger loops will be produced as time increases. As the strings explore the extra dimensions, we expect the winding number to increase with time, so that the minimum length that a cycloop can reach will depend on the time it was formed. (Of course, depending on energetic considerations, a large cycloop of winding number $N$ can break up into $N$ cycloops of single winding.) 


\section{Winding Number}

We can study the dependence of the winding number on time using the Extra-Dimensional VOS model introduced in [16]. In that model there is a parameter $w_{\ell}$, which provides a measure of the amount of string length in the extra compact dimensions. In particular $w_{\ell}^{2}$ is the average value of the ratio of the square of the string length in the $D-3$ compact dimensions, over the square of the length in all dimensions. For a loop of total length $\ell$, the parameter $w_{\ell}$ can be used to estimate its length in the compact dimensions and this can be turned into a winding number, subject to assumptions about the structure of the string in these dimensions and the non-trivial cycles in the compact manifold.

We can identify two distinct scenarios: in the first one, the strings are assumed to form with an initial correlation length $L_{0}$ smaller than the size of the compact dimensions $R$. We suppose they form a random walk shaped-structure in these dimensions, which can give rise to non-trivial windings (see Fig. 1). Having $L_{0}<R$ does not appear to be the usual brane inflation scenario (but see Ref. [21]); presumably, the Brownian strings slowly migrate off the branes because of transverse momenta arising from the brane collision. On the other hand, for $L_{0}>R$, we have the scenario in which straighter strings acquire windings more directly because of their transverse velocities. Since these velocities can only be correlated at length-scales less than the correlation length of the network, the strings begin winding around the compact dimensions, as illustrated in Fig. 2] We will consider each scenario separately.

(i) Random walk: In this regime, we assume that string winding arises from the strings' spatial structure in the compact dimensions, which can be modelled as a random walk of step $\xi_{\ell}$. For a loop of total length $\ell \gg \xi_{\ell}$, its length in the compact dimensions is approximately $\ell w_{\ell}$. Because of the random walk shape of the string, the total displacement in these dimensions is considerably less, namely $\sqrt{\ell w_{\ell} \xi_{\ell}}=\sqrt{\alpha w_{\ell} \xi_{\ell} t}$. If this displacement is greater than the size of 1-cycles present in the compact manifold, R, a non-trivial winding can be developed with a winding number given by

$$
N \simeq \frac{\sqrt{\alpha w_{\ell} \xi_{\ell} t}}{R}
$$

For a cycloop created at $t=t_{i}$, the minimum length to which it can shrink is then

$$
\ell_{\min } \simeq N R \simeq \sqrt{\alpha w_{\ell} \xi_{\ell} t} \equiv \kappa t_{i}^{1 / 2}
$$




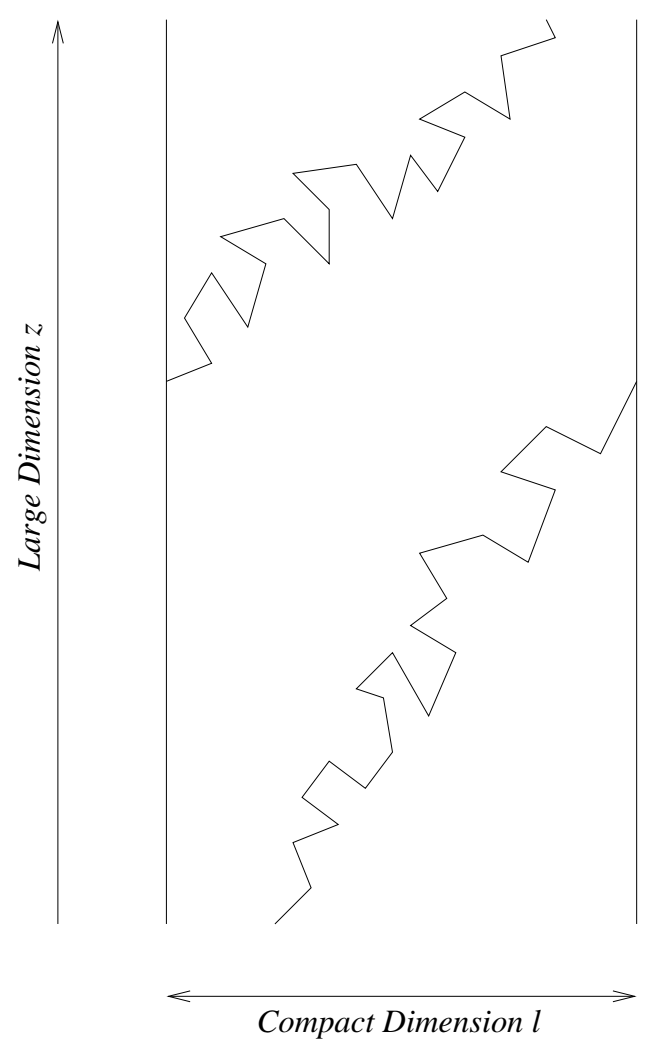

FIG. 1: Random walk regime: The spatial structure of strings in the extra dimension (assumed Brownian) can give rise to non-trivial windings.

where we have set $\kappa \equiv \sqrt{\alpha w_{\ell} \xi_{\ell}}$. Thus, in this regime, the minimum cycloop length is proportional to the square root of the time at which the loop was formed.

(ii) Velocity correlations: We now consider a situation where the strings are produced with significant velocity components in the compact dimension [16]. These cannot be correlated beyond distances of order the correlation length at string formation $L_{0}$, and so points on the string separated by distances greater than $L_{0}$ can be expected to move in different directions. Such motion in the compact dimensions can result in string winding, if the initial correlation length is greater than the size of the non-trivial cycles present in the compact manifold.

To quantify this, we estimate that the number of windings per initial correlation length will be given by

$$
N_{L_{0}}=w_{\ell} \frac{L_{0}}{R}, \quad L_{0}>R
$$

A loop of length $\ell$ will then have an associated winding number

$$
N \simeq \begin{cases}\sqrt{\frac{\ell}{L_{0}}} N_{L_{0}}=w_{\ell} \frac{\sqrt{\ell L_{0}}}{R}, & \ell>L_{0} \\ \frac{\ell}{L_{0}} N_{L_{0}}=w_{\ell} \frac{\ell}{R} & , \quad \ell<L_{0} .\end{cases}
$$



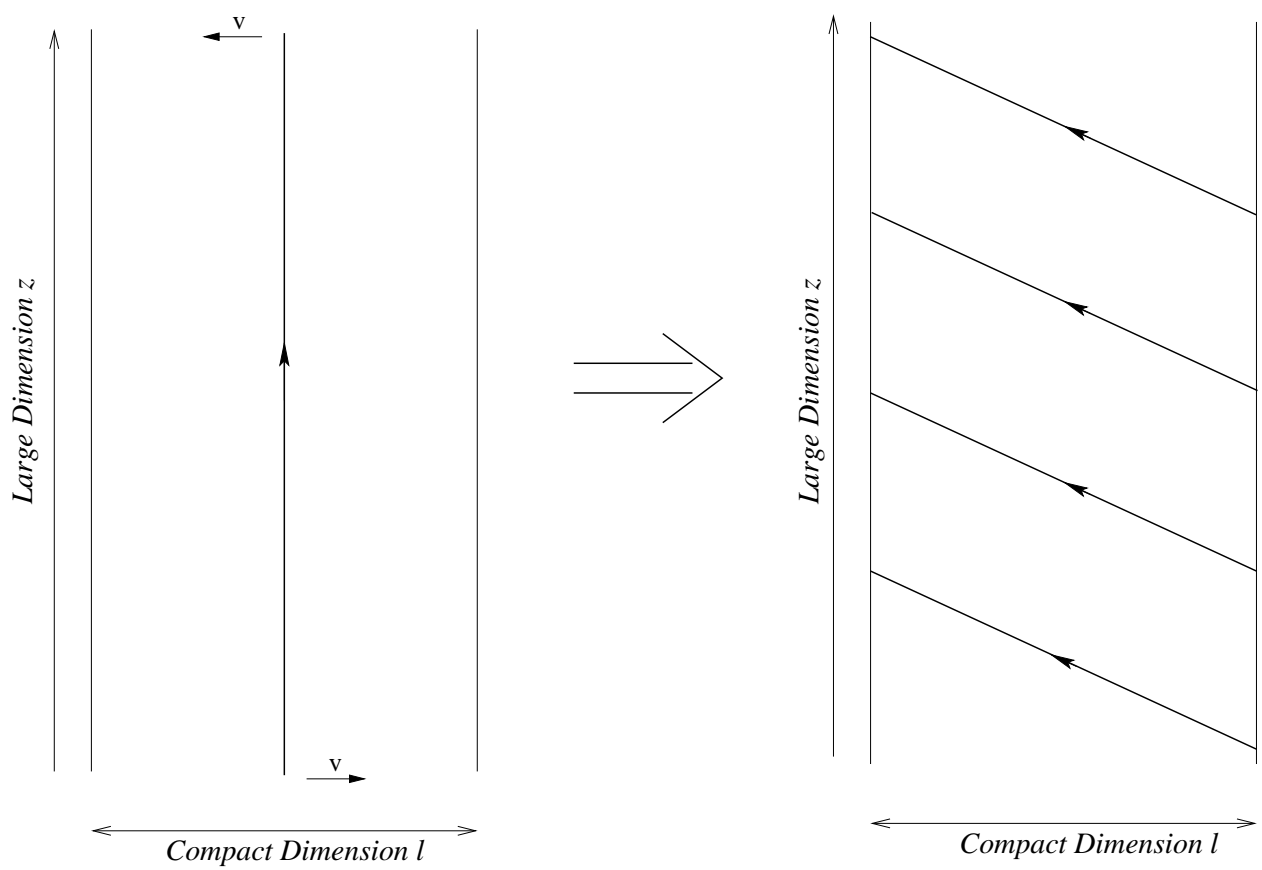

FIG. 2: Velocity correlations regime: String velocities in the extra dimensions cannot be correlated at distances greater than the correlation length at formation $L_{0}$. Thus the velocities at the endpoinds of a string segment of length $L_{0}$ can be expected to have different directions. This would tilt the string as shown to produce windings.

Loops are typically produced at a fraction of the correlation length, so the first loops will have a size $\ell<L_{0}$. Since the correlation length increases with time, loops produced at later times will have a size greater than the initial correlation length at formation. However, the loop number density is, as in the three-dimensional case, dominated by the smallest loops in the distribution (see next section) and so the vast majority of loops will have $\ell<L_{0}$. Writing $\ell=\alpha t$ we obtain for the winding number

$$
N \simeq \frac{w_{\ell} \alpha}{R} t
$$

so that a cycloop created at time $t_{i}$ will reach a minimum length

$$
\ell_{\min } \simeq N R \simeq w_{\ell} \alpha t_{i}
$$

This is linear in loop formation time, in contrast to the previous (random walk) regime where $\ell_{\min } \propto t_{i}^{1 / 2}$. 
It is convenient to introduce a function $f$ such that

$$
\ell_{\min }=f\left(t_{i}\right)=\left\{\begin{array}{l}
\kappa t_{i}^{1 / 2}, \text { random walk regime } \\
w_{\ell} \alpha t_{i}, \text { velocity correlations regime }
\end{array}\right.
$$

Next we study the cycloop number and energy density distribution in both regimes.

\section{Number and Energy Density Distribution}

We first express the number distribution (6) in terms of the cycloop length at time $t$ (equation (6) is expressed in terms of the initial length at production). We recall from last section that the smallest possible cycloops have size $\ell_{\min }\left(t_{c}\right)=f\left(t_{c}\right)$ where $t_{c}$ is the time at which the first cycloops form and the function $f$ is either proportional to a square root or linear, depending on whether the winding is produced by random walk spatial structure or velocity correlations. Also, the largest possible loops at time $t$ are the ones just produced with length $\alpha$. The cycloop distribution is therefore zero outside this range.

At any time $t$ cycloops fall into two classes: those that have reached their minimum size and those that did not have enough time to do so and are still shrinking. At early times all loops in the distribution are shrinking but at some point $t_{c f}>t_{c}$ the first loops of initial size $\alpha t_{c}$ reach their minimum length $\ell_{\min }\left(t_{c}\right)$ and freeze. For $t>t_{c f}$ there are both shrinking and stabilised cycloops in the distribution. Thus, for $t>t_{c f}$ the cycloop number density distribution is

$$
n_{\mathrm{cycl}}(\ell, t)=\left\{\begin{array}{cll}
\frac{g \tilde{c} \gamma^{-3} \alpha^{1 / 2}}{t^{3 / 2}\left[\ell+\Gamma G \mu\left(t_{i f}-t_{i}\right)\right]^{5 / 2}}, & \ell_{\min }\left(t_{c}\right)<\ell<\ell_{\min }\left(t_{m}\right) \\
\frac{g \tilde{c} \gamma^{-3} \alpha^{1 / 2}}{t^{3 / 2}\left[\ell+\Gamma G \mu\left(t-t_{i}\right)\right]^{5 / 2}}, & \ell_{\min }\left(t_{m}\right)<\ell<\alpha t \\
0 & , \quad \ell<\ell_{\min }\left(t_{c}\right) \text { or } \ell>\alpha t
\end{array}\right.
$$

where $t_{i f}$ is the time at which a loop of initial size $\ell_{i}=\alpha t_{i}$ reaches its minimum size $\ell=\ell_{\min }\left(t_{i}\right)$ and freezes. This is uniquely determined by $\ell_{i}=\ell+\Gamma G \mu\left(t_{i f}-t_{i}\right)$ giving

$$
t_{i f}=\frac{(\alpha+\Gamma G \mu) t_{i}-\ell_{\min }\left(t_{i}\right)}{\Gamma G \mu} .
$$

Also the time $t_{m}$, which determines the length where the two distributions in (18) match, is the time of formation of cycloops that have just reached their minimum size $\ell_{\min }\left(t_{m}\right)$ at time $t$. This is found by saturating $t_{i f} \leq t$, which has only one real solution for both functions $\ell_{\min }\left(t_{i}\right)$ discussed above. 
The distribution (18) is dominated by cycloops of smallest initial length, which have already reached their minimum size. Thus it suffices to consider only the frozen cycloops (ignoring all other loops in the distribution), whose number density distribution is given by the first part of equation (18). Noting that the quantity in the square bracket is equal to $\ell_{i}=\alpha t_{i}$ and using equation (17) this becomes

$$
n(\ell, t)=\frac{g \tilde{c} \gamma^{-3}}{\alpha^{2}\left[f^{-1}(\ell)\right]^{5 / 2} t^{3 / 2}}
$$

with $f^{-1}$ the inverse function of $f$, equal to $t_{i}$ by equation (17). We now consider the two winding regimes discussed in last section.

(i) Random walk: In this case $f^{-1}(\ell)=(\ell / \kappa)^{2}$ and equation (20) becomes

$$
n(\ell, t)=\frac{g \tilde{c} \gamma^{-3} \kappa^{5}}{\alpha^{2} \ell^{5} t^{3 / 2}}
$$

Integrating this over all loop sizes yields the number density of cycloops at time $t$

$$
\begin{array}{r}
n(t)=\int_{\ell_{\min }\left(t_{c}\right)}^{\ell_{\min }\left(t_{m}\right)} n(\ell, t) d \ell \simeq \frac{1}{4} \frac{g \tilde{c} \gamma^{-3} \kappa^{5}}{\alpha^{2}\left[\ell_{\min }\left(t_{c}\right)\right]^{4} t^{3 / 2}} \\
=\frac{1}{4} g \tilde{c} \gamma^{-3} \alpha^{-3 / 2} w_{\ell}^{1 / 2} \frac{\xi_{\ell}^{1 / 2}}{t_{c}^{2} t^{3 / 2}}
\end{array}
$$

where we have used the fact that the distribution is dominated by loops of the smallest size so that the integral can be approximated by its lower limit.

The cycloop energy density at time $t$ can be found in a similar way

$$
\begin{aligned}
\rho_{\mathrm{cycl}}(t)=\int_{\ell_{\min }\left(t_{c}\right)}^{\ell_{\min }\left(t_{m}\right)} n(\ell, t) \mu \ell d \ell & \simeq \frac{1}{3} \frac{g \mu \tilde{c} \gamma^{-3} \kappa^{5}}{\alpha^{2}\left[\ell_{\min }\left(t_{c}\right)\right]^{3} t^{3 / 2}} \\
& =\tilde{c} \gamma^{-3} \alpha^{-1} w_{\ell} \frac{\xi_{\ell} \mu}{\left(t_{c} t\right)^{3 / 2}} .
\end{aligned}
$$

Note that this depends on $\xi_{\ell}$, the step of the random walk which models the spatial structure of loops in the extra dimensions.

(ii) Velocity correlations: In this regime we have $f^{-1}(\ell)=\ell /\left(w_{\ell} \alpha\right)$. The number density distribution (20) now reads

$$
n(\ell, t)=\frac{g \tilde{c} \gamma^{-3} \alpha^{1 / 2} w_{\ell}^{5 / 2}}{\ell^{5 / 2} t^{3 / 2}}
$$

The corresponding cycloop number and energy densities at time $t$ are now

$$
n(t) \simeq \frac{2}{3} \frac{g \tilde{c} \gamma^{-3} \alpha^{1 / 2} w_{\ell}^{5 / 2}}{\left[\ell_{\min }\left(t_{c}\right)\right]^{3 / 2} t^{3 / 2}}=\frac{2}{3} g \tilde{c} \gamma^{-3} \alpha^{-1} w_{\ell} \frac{1}{\left(t_{c} t\right)^{3 / 2}}
$$


and

$$
\rho_{\text {cycl }}(t) \simeq 2 \frac{g \mu \tilde{c} \gamma^{-3} \alpha^{1 / 2} w_{\ell}^{5 / 2}}{\left[\ell_{\min }\left(t_{c}\right)\right]^{1 / 2} t^{3 / 2}}=2 \tilde{c} \gamma^{-3} w_{\ell}^{2} \frac{\mu}{t_{c}^{1 / 2} t^{3 / 2}} .
$$

In this case the cycloop energy density does not depend on the parameter $\alpha$. Since $\alpha$ is proportional to $\gamma$ this leads to a different dependence of the cycloop energy density on the effective intercommuting probability $P_{\text {eff }}$. The dependence on $w_{\ell}$ and $t_{c}$ is also different. These differences will be of significant importance in the next section.

In both regimes the energy density of cycloops scales like matter in the radiation era. This immediately poses a potential monopole problem (or in even closer analogy a vorton problem [31, 32]) and requiring consistency with standard cosmology would constrain models that allow cycloops. We consider such constraints in the following section.

\section{COSMOLOGICAL CONSTRAINTS}

We have seen that the winding number of cycloops increases monotonically with time, so loops produced at later times will have more windings and hence more length. However, most loops are produced at earlier times, when the density of the long string network is higher, and so the cycloop energy density is dominated by cycloops of the smallest possible winding (21-26). In the usual scenario where the correlation length at the time of cosmic string formation is much greater than the size of the extra dimensions $\left(L_{0} \gg R\right)$, even the smallest cycloops can have a large winding number. However, if the correlation length at string formation is less than the compactification scale (as in Ref. [21]), the first closed loops formed by long string interactions will have no windings and will eventually decay. The cycloop energy density will be dominated by loops with winding number of order one, produced at later times, when the spatial structure will have developed enough to allow non-trivial windings.

To quantify the cosmological constraints imposed by cycloops we consider a simple D5 $\overline{\mathrm{D} 5}$ inflation model in which two of the dimensions parallel to the branes are compactified on a torus of size $R$. The branes collide and annihilate, producing D3 and $\overline{\mathrm{D} 3}$ branes, which, if they wrap the same dimensions as the mother branes, can be seen as cosmic strings.

We consider the 'usual' case where the correlation length is of order $L_{0} \sim H^{-1} \simeq M_{p l} / M_{s}^{2}$, with the string scale $M_{s}$ set to a low GUT scale by the CMB data [4, 8] and the compactification size $R \sim 10 M_{s}^{-1}$. Since the correlation length is much greater than $R$, the Kibble 
mechanism only takes place in the large three dimensions, so the 3-branes produced must wrap the same compact volume as their mother branes [4]. We can therefore consider them as strings and ignore their internal compact dimensions. However, there are also compact dimensions transverse to the branes, in the bulk where the strings can move. Long string interactions can therefore produce cycloops wrapping around these dimensions.

We consider the two winding regimes of section IB 1 separately:

(i) Random walk: Here the cycloop winding number is determined by (random walk shaped) spatial structure in the extra dimensions, and the cycloop energy density as a function of formation time is given by equation (23). To account for changes in the effective number of degrees of freedom near mass thresholds during the evolution of the early universe, we make use of the entropy density of relativistic species

$$
s(T)=\frac{2 \pi^{2}}{45} \mathcal{N}_{s}(T) T^{3}
$$

where $\mathcal{N}_{s}(T)$ is the effective number of relativistic degrees of freedom for entropy as a function of the background plasma temperature $\mathrm{T}$.

Neglecting annihilations between cycloops of opposite winding, the ratio of the number density of cycloops to the total entropy density is conserved, so that we can write for the cycloop energy density

$$
\rho_{\text {cyc }}(T)=\mu \int n\left(\ell, t_{f}\right) \ell d \ell \frac{s(T)}{s\left(T_{f}\right)}=\mu \int n\left(\ell, t_{f}\right) \ell d \ell \frac{\mathcal{N}_{s}(T)}{\mathcal{N}_{s}\left(T_{f}\right)}\left(\frac{T}{T_{f}}\right)^{3}
$$

where $t_{f}$ (resp. $T_{f}$ ) denotes the time (resp. temperature) at which the first cycloops, produced at time $t_{c}$, freeze to their minimum size. Note that we assumed no significant change in the effective number of degrees of freedom between times $t_{c}$ and $t_{f}$.

Writing the energy density of relativistic species as

$$
\rho_{\mathrm{rad}}(t)=\frac{\pi^{2}}{30} \mathcal{N}(T) T^{4}
$$

where $\mathcal{N}(T)$ is the effective number of relativistic degrees of freedom for energy density, we form the ratio of $\rho_{\text {cyc }}$ to the critical density of the universe $\rho_{\text {crit }}$ at matter-radiation equality, finding

$$
\frac{\rho_{\text {cyc }}\left(T_{\text {eq }}\right)}{\rho_{\text {crit }}\left(T_{\text {eq }}\right)}=\frac{1.48}{\pi^{2}} g \tilde{c} \gamma^{-3} \alpha^{-1} w_{\ell} \mathcal{N}_{s}\left(T_{s}\right)^{1 / 2} \frac{\mathcal{N}_{s}\left(T_{\text {eq }}\right)}{\mathcal{N}\left(T_{\text {eq }}\right)}\left(\frac{\mathcal{N}_{s}\left(T_{s}\right)}{\mathcal{N}_{s}\left(T_{f}\right)}\right)^{1 / 4}\left(\frac{\xi_{\ell} \mu T_{s}^{3}}{T_{\text {eq }} M_{p l}^{3}}\right)
$$


Here we have used equation (23) and assumed instantaneous reheating to a radiation dominated universe immediately after the end of inflation, so that cosmic time can be related to the plasma temperature $T$ by

$$
t=1.51 \mathcal{N}_{s}(T)^{-1 / 2} \frac{M_{p l}}{T^{2}}
$$

where $M_{p l}=2.44 \times 10^{18} \mathrm{GeV}$ is the reduced Planck mass, related to the Planck mass $m_{p l}=1.22 \times 10^{19} \mathrm{GeV}$ by $m_{p l}=\sqrt{8 \pi} M_{p l}$. We have also assumed that the first cycloops are formed at the time of cosmic string formation $t_{s}$ (just after brane collision and the end of inflation) so that we can write $T_{c} \simeq T_{s}$, which is approximately equal to the reheating temperature.

A constraint on the model can be imposed by requiring that cycloops do not dominate the energy density of the universe before the time of equal matter-radiation $t=t_{\text {eq }}$ that is, demanding

$$
\frac{\rho_{\text {cyc }}\left(T_{\text {eq }}\right)}{\rho_{\text {crit }}\left(T_{\text {eq }}\right)} \leq 1
$$

For typical models the string tension $\mu$ is of order the string scale $M_{s}$ squared (see for example [5]) and assuming instantaneous reheating we have $M_{s} \sim T_{s}$. In the random walk scenario, the stepsize $\xi_{\ell}$ in the compact dimension cannot be smaller than the inverse string scale or larger than the size of the compact dimension $R \sim 10 M_{s}^{-1}$. Thus we have to assume $\xi_{\ell} \sim M_{s}^{-1} \sim T_{s}^{-1}$. We can also take $\mathcal{N}_{s}\left(T_{s}\right)$ of order $10^{3}$, as in some GUT models. We will take the effective intercommuting probability to lie in the range $10^{-3}<P_{\text {eff }}<1$. (Note that strictly speaking it is the actual probability $P$ which spans this range [12], so the corresponding range for $P_{\text {eff }}$ depends on the functional form $P_{\text {eff }}=f(P)$, which is still uncertain.)

Usual field theory strings have typically $\tilde{c} \sim 1$ but, as discussed above, in our setup $\tilde{c}$ is of order the effective intercommuting probability $P_{\text {eff }}$. The extra-dimensional VOS model 16 ] suggests $\gamma \sim \tilde{c} \sim P_{\text {eff }}$. Numerical simulations of string networks provide evidence that loop production is typically peaked at sizes of order $10^{-2}-10^{-3}$ the correlation length [26, 27]. Thus we will assume $\alpha \sim 10^{-2} \gamma \sim 10^{-2} P_{\text {eff }}$. Note however, that this assumption is only valid once the long string network has reached a scaling regime. If cycloops are produced early enough, it may well be that the network has not yet achieved scaling, in which case $\alpha$ can be considerably larger (we will return to this point later). From numerical simulations, the Lorentz factor $g$ can be taken to be $g \simeq 1 / \sqrt{2}[25]$. 
Finally, we need to choose some value for the parameter $w_{\ell}$, which, as mentioned above, is a measure of the relative amount of string length in the extra dimensions. Unfortunately, this is somewhat uncertain and as yet not fully understood since this parameter is determined by the nature of the brane collision. For example if the collision is violent, one expects the strings formed to have significant velocities in the extra dimensions, which would force them to explore these dimensions. On the other hand, a perfectly adiabatic collision would give rise to smooth strings lying on the plane of brane collision. We will take $w_{\ell} \sim 0.1$, corresponding to strings with approximately one tenth of their length in the extra dimensions.

With this choice of parameters and for $P_{\text {eff }} \simeq 10^{-2}$ the bound (32) becomes

$$
10^{8} \frac{T_{s}^{4}}{T_{\mathrm{eq}} M_{p l}^{3}} \leq 1 \Longrightarrow T_{s} \leq\left(10^{-8} T_{\mathrm{eq}} M_{p l}^{3}\right)^{1 / 4} \sim 10^{9} \mathrm{GeV}
$$

This is orders of magnitude lower than the typical energy scale of these unwarped brane inflation models (GUT scale) and thus such models would be ruled out, unless some other mechanism (e.g. a short period of subsequent inflation [33]) operates to reduce the cycloop energy density.

(ii) Velocity correlations: In this case the energy density of cycloops is given by equation (26). Working as before we find

$$
\frac{\rho_{\text {cyc }}\left(T_{\text {eq }}\right)}{\rho_{\text {crit }}\left(T_{\text {eq }}\right)}=\frac{13.3}{\pi^{2}} g \tilde{c} \gamma^{-3} w_{\ell}^{2}\left(\frac{\mathcal{N}_{s}\left(T_{s}\right)}{\mathcal{N}_{s}\left(T_{f}\right)}\right)^{1 / 4} \frac{\mathcal{N}_{s}\left(T_{\text {eq }}\right)}{\mathcal{N}\left(T_{\text {eq }}\right)}\left(\frac{\mu T_{s}}{T_{\text {eq }} M_{p l}^{2}}\right)
$$

leading to the constraint (again with typical choice of parameters)

$$
10^{2} \frac{T_{s}^{3}}{T_{\mathrm{eq}} M_{p l}^{2}} \leq 1 \Longrightarrow T_{s} \leq\left(10^{-2} T_{\mathrm{eq}} M_{p l}^{2}\right)^{1 / 3} \sim 10^{8.5} \mathrm{GeV}
$$

It is interesting to comment on the differences between the constraints (33) and (35). In the random walk regime (33) the temperature which saturates the bound scales like $\left(T_{\text {eq }} M_{p l}^{3}\right)^{1 / 4}$, resembling the usual magnetic monopole constraint. On the other hand, the corresponding scaling in the velocity correlation regime is $\left(T_{\text {eq }} M_{p l}^{2}\right)^{1 / 3}$, which by itself would produce a stronger bound by approximately two orders of magnitude. However, some of this difference is compensated by the different numerical coefficients in (33) and (35).

Indeed, comparing equations (30) and (34) we observe that in the velocity correlation regime there is no dependence on $\alpha$. Also, $w_{\ell}$ appears quadratically rather than linearly, while the dependence on $\mathcal{N}_{s}\left(T_{s}\right)$ is essentially lost. Note that because of the quartic (resp. cubic) root in equation (33) (resp. (35)) the cycloop constraint is not strongly sensitive on 
the choice of parameters. However, different choices of $P_{\text {eff }}$ in the range $10^{-3}<P_{\text {eff }}<1$ and $w_{\ell}$ in the range $10^{-2}<w_{\ell}<1$ can lead to changes in $\left(T_{s}\right)_{\min }$ of up to a factor of $10^{2}-10^{3}$.

Thus in most of the parameter space, the cycloop constraint rules out brane inflation models with a low GUT energy scale or higher that produce stable cosmic strings and allow non-trivial cycles in their compact manifolds. However, there is a small region in the parameter space, corresponding to $P_{\text {eff }} \simeq 1, \alpha \sim \gamma$ and $w_{\ell}<10^{-2}$ (in the random walk regime), which is still consistent with an energy scale of order $10^{12} \mathrm{GeV}$. One could imagine such a model arising from a quasi-adiabatic brane collision (thus justifying a small value of $\left.w_{\ell}\right)$ in which the cycloops are produced before scaling has been reached (hence allowing a large value of $\alpha$ ). In such a model the cycloop could play the role of dark matter.

Finally, we consider the recently proposed scenario [21] that the correlation length at string formation is less than the size of the extra dimensions. In that case the 3-branes produced do not have to wrap the compact dimensions so domain wall-like objects can be formed. One expects that these objects would interact to produce strings and indeed toy model simulations in Ref. [21] support this. It is also noted that monopole-like objects, namely D1-branes wrapping the compact dimensions can be formed as well.

It is clear that the cycloops we considered above, i.e. the closed loops (winding around compact dimensions) produced by long string network interactions, cannot be created soon after the end of inflation in the case of $L_{0} \ll R$, simply because the correlation length is far too small to allow their formation. The first cycloops will form when the correlation length becomes much greater than the size of the extra dimensions or more specifically, in the random walk regime, when the displacement of loops in these dimensions $\kappa t^{1 / 2}$ becomes greater than the compactification scale $R$ (note that velocity correlations for $R>L_{0}$ can only give rise to winding in a statistical sense, in which case we recover the random walk regime). Thus, the considerations presented above would apply for cycloops produced at a time $t_{c}$, much later than the end of inflation, when the string network density will be smaller. This leads to a smaller cycloop number density albeit with a larger average winding number. The net result is that our considerations constrain (mostly) $T_{c}$ (i.e. $T$ at cycloop formation) rather than $T_{s}$ ( $T$ at string formation and reheating), so the constraint on $T_{s}$ itself is weaker. Again, this opens up the possibility that the cycloop can provide a dark matter candidate. Also note that in a realistic model, the scalar fields corresponding to the string position in the extra dimensions should be stabilised at late times [12], so cycloop production may not 
be possible if this localisation takes place at $t<t_{c}$.

\section{CONCLUSIONS}

We have considered cosmic string evolution in the presence of compact extra dimensions and focused on the possibility that long string interactions can produce closed loops wrapping around compact dimensions. In the case that the compact manifold admits non-trivial 1cycles, these loops can become topologically trapped and behave like stable monopoles or, in closer analogy, vortons.

We have studied the dependence of the winding number of such a loop on the time $t$ when the loop was formed and identified two distinct regimes: one in which windings arise as a result of (random walk shaped) spatial structure in the compact dimensions, and one where they are created by string velocity correlations in these dimensions. In the former case the winding number is proportional to $t^{1 / 2}$, while in the latter to $t$. In both cases, we studied the evolution of these objects (dubbed cycloops) and we have shown that their energy density scales like matter in the radiation era, the distribution being dominated by cycloops of the smallest possible winding number, produced at the earliest possible times. This immediately gives rise to a potential monopole problem, which can be used to impose constraints on models that allow cycloops.

Considering these constraints in the case of simple (unwarped compactification) brane inflation models and assuming instantaneous reheating immediately after brane collision, we find that in the majority of the parameter space the energy scale of inflation is required to be less than $10^{10} \mathrm{GeV}$. Since the typical scale of these models is a low GUT scale, this constraint rules out these models unless some mechanism (e.g. a secondary inflationary phase [33]) is provided to dilute the cycloop density. There is however a small region in the parameter space, corresponding to an intercommuting probability of order unity and large loops (such large loops could be produced at very early times when the loop size is determined by the initial conditions rather than the asymptotic scaling behaviour of the network), which may still be consistent with low GUT scale brane inflation. In such a model the cycloop may be an attractive dark matter candidate. Another situation in which the cycloops are allowed as dark matter is if they are formed long after the brane collision, in which case the cycloop constraint is alleviated. 
We stress that our analysis directly constrains only models which allow non-trivial 1cycles in their compact manifolds (e.g. toroidal compactification), but not, for example, the simplest warped-compactification models with simply-connected throats (see however

Ref. [34], where stable monopole solutions were found in the conifold geometry). The outstanding uncertainty in these estimates remains the dynamics of the brane collision and ensuing string-forming transition.

\section{Acknowledgments}

We are grateful to Carlos Martins for many discussions. A.A. is funded by EPSRC, the Cambridge European Trust and the Cambridge Newton Trust. E.P.S.S. is supported by PPARC, grant number PP/C501676/1.

As this work was completed, a replacement version of hep-ph/0412290 was put on the archive. This new version appears to contain some overlapping results for our relic loop density, though that discussion seems to depend on localising potentials in the extra dimensions.

[1] T. W. B. Kibble (2004), astro-ph/0410073.

[2] G. Dvali and S. H. H. Tye, Phys. Lett. B450, 72 (1999), hep-ph/9812483.

[3] C. P. Burgess, M. Majumdar, D. Nolte, F. Quevedo, G. Rajesh, and R.-J. Zhang, JHEP 0107, 047 (2001), hep-th/0105204.

[4] S. Sarangi and S. H. H. Tye, Phys. Lett. B536, 185 (2002), hep-th/0204074.

[5] G. Dvali and A. Vilenkin, JCAP 0403, 010 (2004), hep-th/0312007.

[6] E. J. Copeland, R. C. Myers, and J. Polchinski, JHEP 06, 013 (2004), hep-th/0312067.

[7] N. T. Jones, H. Stoica, and S. H. H. Tye, Phys. Lett. B563, 6 (2003), hep-th/0303269.

[8] L. Pogosian, S. H. H. Tye, I. Wasserman, and M. Wyman, Phys. Rev. D68, 023506 (2003), hep-th/0304188.

[9] T. Damour and A. Vilenkin (2004), hep-th/0410222.

[10] J. Polchinski (2004), hep-th/0410082.

[11] J. Polchinski (2004), hep-th/0412244. 
[12] M. G. Jackson, N. T. Jones, and J. Polchinski (2004), hep-th/0405229.

[13] C. J. A. P. Martins and E. P. S. Shellard, Phys. Rev. D53, 575 (1996), hep-ph/9507335.

[14] C. J. A. P. Martins and E. P. S. Shellard, Phys. Rev. D54, 2535 (1996), hep-ph/9602271.

[15] C. J. A. P. Martins and E. P. S. Shellard, Phys. Rev. D65, 043514 (2002), hep-ph/0003298.

[16] A. Avgoustidis and E. P. S. Shellard (2004), hep-ph/0410349.

[17] C. J. A. P. Martins, Phys. Rev. D70, 107302 (2004), hep-ph/0410326.

[18] S. H. H. Tye, I. Wasserman, and M. Wyman (2005), astro-ph/0503506.

[19] M. Sakellariadou and A. Vilenkin, Phys. Rev. D42, 349 (1990).

[20] M. Sakellariadou (2004), hep-th/0410234.

[21] N. Barnaby, A. Berndsen, J. M. Cline, and H. Stoica (2004), hep-th/0412095.

[22] T. Matsuda (2004), hep-ph/0412290.

[23] E. Babichev and M. Kachelriess, Phys. Lett. B614, 1 (2005), hep-th/0502135.

[24] T. Vachaspati and A. Vilenkin, Phys. Rev. D30, 2036 (1984).

[25] A. Vilenkin and E. P. S. Shellard, Cosmic Strings and Other Topological Defects (Cambridge University Press, 1994).

[26] D. Bennett and F. Bouchet, Phys. Rev. D41, 2408 (1990).

[27] B. Allen and E. P. S. Shellard, Phys. Rev. Lett. 64, 119 (1990).

[28] V. Vanchurin, K. Olum, and A. Vilenkin (2005), gr-qc/0501040.

[29] T. W. B. Kibble, Nucl. Phys. B252, 227 (1985).

[30] A. Avgoustidis and E. Shellard (in preparation).

[31] C. J. A. P. Martins and E. P. S. Shellard, Phys. Rev. D57, 7155 (1998), hep-ph/9804378.

[32] C. J. A. P. Martins and E. P. S. Shellard, Phys. Lett. B445, 43 (1998), hep-ph/9806480.

[33] C. P. Burgess, R. Easther, A. Mazumdar, D. F. Mota, and T. Multamaki (2005), hepth/0501125.

[34] J. J. Blanco-Pillado and A. Iglesias (2005), hep-th/0504068. 